\title{
Acute pulmonary embolism in COVID-19 related hypercoagulability
}

\author{
Cerruti Lorenzo $^{1} \cdot$ Boscaro Francesca $^{1} \cdot$ Poletto Francesco $^{1} \cdot$ Campello Elena $^{1} \cdot$ Spiezia Luca $^{1}{ }^{\mathbb{D}} \cdot$ Simioni Paolo $^{1}$
}

Published online: 30 May 2020

○) Springer Science+Business Media, LLC, part of Springer Nature 2020

\begin{abstract}
Since December 2019, a novel Coronavirus (SARS-CoV-2) was confirmed as the etiologic agent of a worldwide outbreak of a pneumonia that can result in severe respiratory failure. This clinical entity seems to be associated with a marked hypercoagulable state that causes both arterial and venous thromboembolic complications. Therefore, an adequate anti-thrombotic prophylaxis is recommended in hospitalized COVID-19 patients. Although rapidly worsening respiratory symptoms in a patient with SARS-CoV-2 respiratory infection may correlate with worsening pneumonia itself, it may also mask a pulmonary embolism. We report the case of a 50-year-old man affected by SARS-CoV-2 pneumonia, who developed acute pulmonary embolism.
\end{abstract}

Keywords SARS-CoV-2 $\cdot$ COVID-19 $\cdot$ Coagulation $\cdot$ Interstitial pneumonia $\cdot$ Pulmonary embolism $\cdot$ Case report

\section{Highlights}

- We reported a case of pulmonary embolism precipitating worsening respiratory failure in a 50-year-old man with COVID-19 pneumonia.

- Whole blood thromboelastometry profiles indicated that the patient was indeed in a severe hypercoagulable state.

- Thromboelastometry could be a useful tool to screen for COVID-19 related hypercoagulability and to determine the optimal anticoagulant treatment.

\section{Background}

Coronaviruses (CoVs) are enveloped, single-stranded RNA viruses that can cause respiratory, enteric and neurologic symptoms in humans [1]. In December 2019, a novel CoV caused an outbreak of severe pneumonia in Wuhan (China)

Electronic supplementary material The online version of this article (https://doi.org/10.1007/s11239-020-02160-1) contains supplementary material, which is available to authorized users.

Spiezia Luca

luca.spiezia@unipd.it

1 General Internal Medicine and Thrombotic and Haemorrhagic Diseases Unit, Department of Medicine, Padova University Hospital, Padua, Italy and quickly spread worldwide soon thereafter. This clinical entity was later defined by the World Health Organization as Coronavirus Disease 2019 (COVID-19) and its etiologic agent was named Severe Acute Respiratory Syndrome Coronavirus 2 (SARS-CoV-2) [2]. Zhou et al. identified that older age, D-dimer levels $>1 \mu \mathrm{g} / \mathrm{mL}$, and higher SOFA score on admission were associated with higher odds of in-hospital death [3]. Our recent findings revealed that most severe COVID-19 patients-admitted to the Intensive Care Unit for respiratory failure-present a predominant hypercoagulable state that could lead to arterial and venous thromboembolic complications [4]. It has been reported that the infection causes endothelial dysfunction and an inflammatory state that results in increased thrombin generation and reduced fibrinolysis [5]. Furthermore, hypoxia from severe pneumonia may also promote thrombosis, resulting in higher blood viscosity [6]. The presence of occlusive microthrombi in pulmonary small vessels in lung dissection of critical patients appear to confirm these findings [7]. Microcirculation thrombosis and fibrin deposition in alveolar and interstitial lung spaces as final step of the coagulative cascade, may contribute to the acute respiratory failure observed in COVID-19 patients. Hence the reason why an expert consensus in China proposed the implementation of thromboprophylaxis in severe COVID-19 patients [8]. These pathophysiological findings contribute to increase the thrombotic risk in COVID-19 patients, which is already high due to other, known risk factors for thromboembolism 
such as hospitalization, bed rest, corticosteroid therapy, etc. Currently there is limited data on venous thromboembolic complications in COVID-19 patients and its prevalence is probably underestimated. In this regard, it is reasonable to believe that thromboembolic events observed in COVID19 could mirror previously reported data in SARS patients (e.g. deep vein thrombosis $20.5 \%$ and pulmonary embolism 11.4\%) [9].

We report the case of a 50-year-old male, who developed acute pulmonary embolism (PE) due to acute SARS-CoV-2 infection.

\section{Case report}

In early March 2020, a 50-year-old Caucasian male was admitted to the Emergency department of Padova University Hospital, Italy for acute chest pain associated with exertional dyspnoea. The patient's clinical history revealed ongoing treatment for hypertension with amiloride/hydrochlorothiazide. He reported no previous thrombotic events or family history of thromboembolism. Two weeks before admission, the patient reported fever $\left(38^{\circ} \mathrm{C}\right)$ associated with cough and weakness. A nasopharyngeal swab performed at home tested positive for COVID-19. The patient was therefore isolated at home, and clinically monitored by his General Practitioner. In the 10 days following the onset of symptoms, his clinical conditions gradually improved, and fever and cough almost completely disappeared without any antibiotic or antiviral therapy. Thirteen days after COVID-19 diagnosis, the patient reported exertional dyspnoea and pleuritic chest pain, associated with painful left leg cramps, redness and tenderness. The patient went to the Emergency Department and thereupon was admitted to the Department of Medicine. At clinical evaluation, he appeared haemodynamically stable with blood pressure $126 / 80 \mathrm{mmHg}$, heart rate $60 \mathrm{bpm}$, with slightly reduced oxygen saturation on room air (93\%). The chest, cardiac and abdominal examinations yielded no relevant findings, whereas his left calf edematous. A room air blood-gas analysis showed moderate hypoxic and hypocapnic respiratory failure $\left(\mathrm{pH} 7.48, \mathrm{pO}_{2} 71 \mathrm{mmHg}\right.$, $\mathrm{pCO}_{2} 35 \mathrm{mmHg}, \mathrm{pO}_{2} / \mathrm{FiO}_{2}$ ratio 339.52). Blood tests at admission revealed mild anaemia ( $\mathrm{Hb} 136 \mathrm{~g} / \mathrm{L})$, leucopaenia $\left(2.50 \times 10^{9} / \mathrm{L}\right)$ and mild thrombocytopaenia $\left(139 \times 10^{9} / \mathrm{L}\right)$ most likely due to the viral infection; D-dimer was elevated $(2625 \mu \mathrm{g} / \mathrm{L})$, as were LDH (492 U/L) and C-Reactive Protein $(67 \mathrm{mg} / \mathrm{L})$. A bed side compression ultrasound (CUS) of the legs revealed a left posterior tibial vein DVT. Clinical suspicion of PE prompted a chest Computed Tomography Angiography that confirmed the presence of left lobar, segmentary and sub-segmentary pulmonary thromboembolism (Fig. 1a), as well as signs of bilateral interstitial pneumonia (Fig. 1b). Additional tests to assess the severity of PE (e.g. cardiac troponin I, BNP levels) were normal; the electrocardiogram was normal and echocardiography did not show significant enlargement of right cardiac chambers nor other significant alterations. The patient's PESI score was 60 , compatible with a low risk of mortality. Other biochemical tests, including renal and liver function indices, electrolytes, traditional coagulation parameters were normal (see Supplementary Table 1). Among microbiological tests, a second nasopharyngeal swab for SARS-CoV-2 and a serological test for Mycoplasma pneumoanie (IgM and $\mathrm{IgG}$ ) were both positive. Serological tests for Streptococcus pneumoniae, Legionella pneumophila and Chlamydia pneumoniae were all negative. Thrombophilic conditions-antithrombin, protein $\mathrm{S}$ and $\mathrm{C}$ deficiency; factor $\mathrm{V}$ Leiden and prothrombin variants; antiphospholipid antibodies-were absent. Whole blood thromboelastometry profiles using a ROTEM ${ }^{\circledR}$ sigma apparatus (Instrumentation Laboratory-Werfen, Barcelona, Spain), showed marked hypercoagulability characterized by shorter Clot Formation Time in INTEM and EXTEM and higher MCF in INTEM, EXTEM and FIBTEM vs. normal range (Fig. 2).

The patient received anticoagulant therapy initially with low molecular weight heparin (LMWH), later switched to oral anticoagulation with apixaban. The patient received a combination of azithromycin and hydroxychloroquine to treat pneumonia, as per hospital protocol for COVID-19 inpatients. Due to a concomitant Mycoplasma infection, the patient also received levofloxacin.

During the first days of hospitalization, the patient required low-flow oxygen therapy with nasal cannulas, later discontinued. Before discharge, we performed a room air six-min walking test which was negative for both dyspnoea onset and oxygen desaturation. The clinical course of our patient was favourable: he was discharged after 10 days of hospitalization with ongoing anticoagulant treatment and a scheduled follow-up at a coagulation Centre after 1 month.

\section{Discussion and conclusions}

The present case report appears to confirm once again the role of severe infections as precipitants of venous thromboembolism, and possibly the key role of the association between SARS-CoV-2 and Mycoplasma in causing a prothrombotic state. The clinical presentation of calf pain with oedema and erythema, exertional dyspnea associated with pleuritic chest pain did not leave any diagnostic doubt. Nevertheless, the clinical presentation of thromboembolic events in COVID-19 patients may not always be as unequivocal. Rapidly worsening respiratory symptoms or sudden hypoxic respiratory failure may stem from worsening pneumonia itself, but may also be attributable to an undiagnosed PE. Therefore, other diagnostic considerations in 
Fig. 1 a A chest computed tomography angiography revealed the presence of a left lobar, segmentary and sub-segmentary PE. b Signs of bilateral, interstital pneumonia at chest computed tomography performed in suspicion of pulmonary embolism

\section{A}
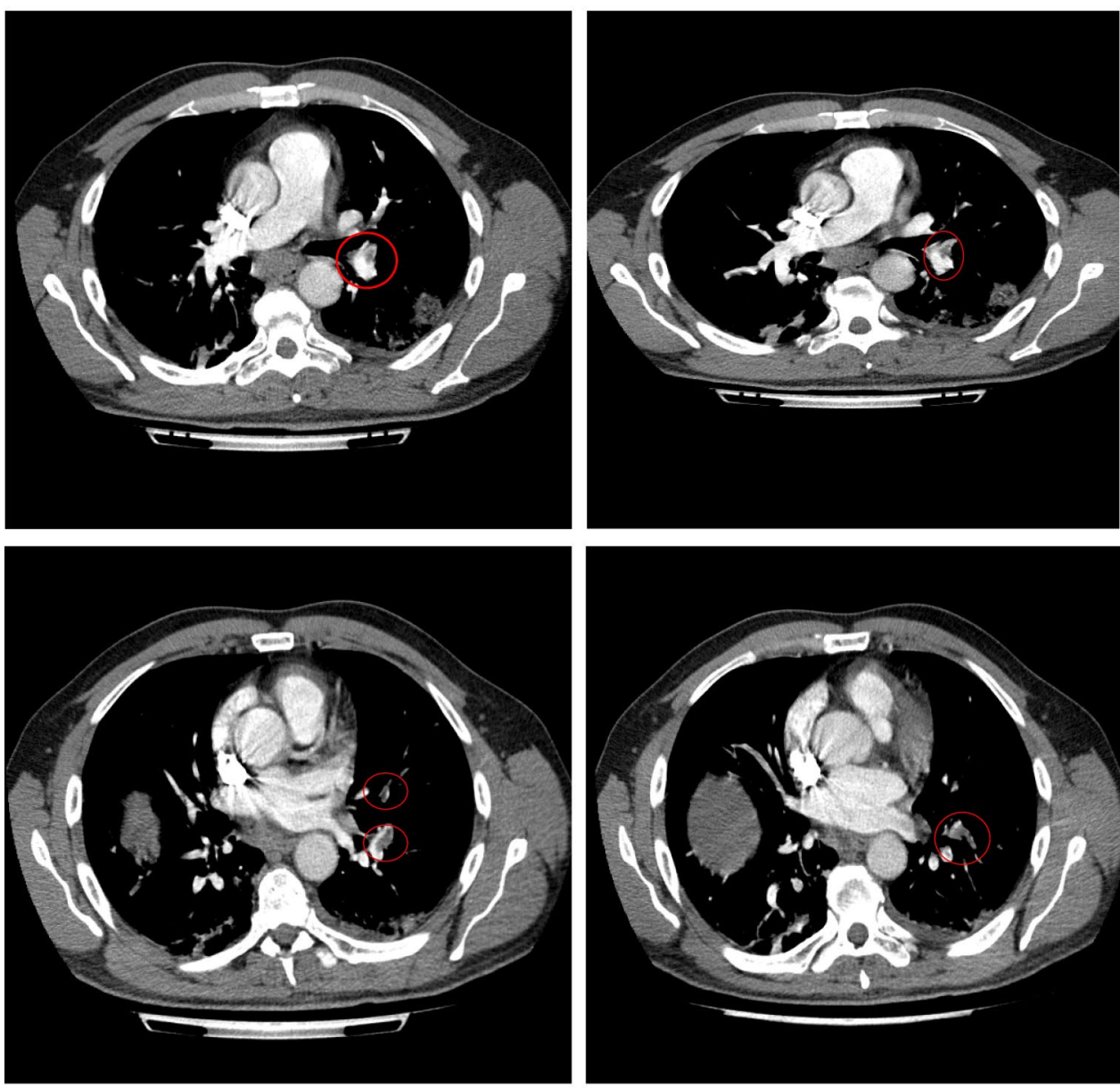

B

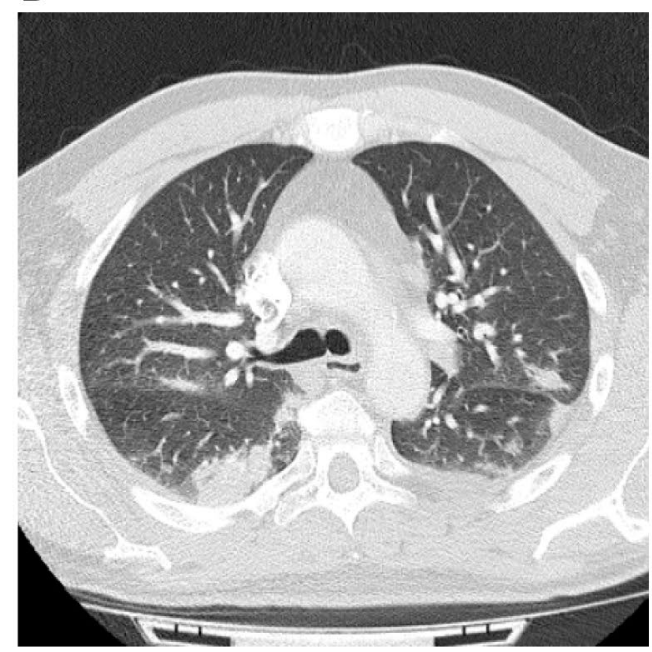

patients with COVID-19 must include PE, considering the high thrombotic risk associated with this infection reported in the literature, and confirmed by our report. The evaluation of the pre-test probability of PE through scores such as Wells score is difficult in these patients, as it may already be high at time of admission. Similarly, elevated D-dimer levels would be non-specific in this setting, thus hindering the diagnostic process. Another confounding factor is that COVID-19 patients often show right ventricular dysfunction on echocardiography irrespective of whether or not they have a PE, hence diagnostic imaging cannot be performed. These observations underline the importance of raising a 

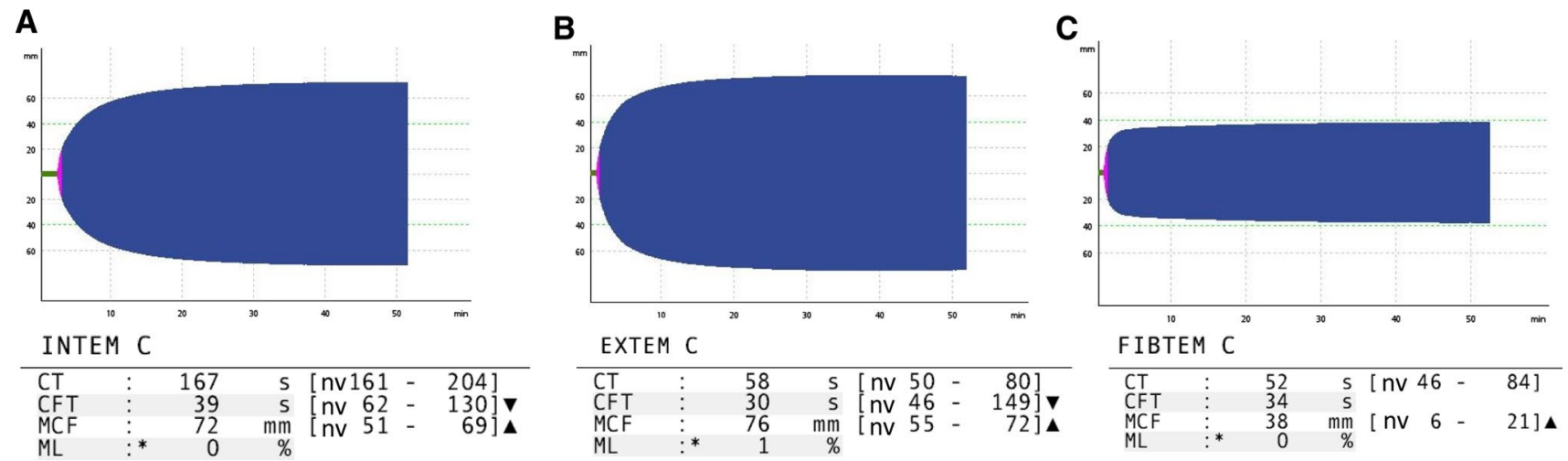

Fig. 2 Patient thromboelastometry profiles. a INTEM test, b EXTEM test, $\mathbf{c}$ FIBTEM test. $C T$ clotting time, $C F T$ clot formation time, $M C F$ maximum clot firmness, $M L$ maximum lysis, $n v$ normal values

clinical suspicion of thromboembolism in presence of worsening dyspnoea and initiating an adequate and timely anticoagulant prophylaxis/therapy in COVID-19 patients, either pharmacological with LMWH or fondaparinux or mechanical in high bleeding risk patients [10].

Another interesting issue raised by our case report is that thromboelastometry could be a worthwhile test to evaluate COVID-19 related hypercoagulability. The main limitation of our study regarding the association between hypercoagulable state observed at the thromboelastogram and the thromboembolic event experienced by our patient is the lack of a thromboelastometric tracing performed at baseline or after discontinuation of anticoagulant treatment. Nevertheless, if our results were to be confirmed by subsequent studies, the thromboelastogram could be considered as a test capable of screening COVID-19 related hypercoagulability. This would allow to identify patients at greatest risk of thrombosis who may benefit from a prophylaxis with a higher dosage than normally suggested as thromboprophylaxis in acute medical conditions.

\section{References}

1. Singh SK (2016) Middle east respiratory syndrome virus pathogenesis. Semin Respir Crit Care Med 37:572-577

2. Phelan AL, Katz R, Gostin LO (2020) The novel coronavirus originating in Wuhan, China: challenges for global health governance. JAMA. https://doi.org/10.1001/JAMA.2020.1097
3. Zhou F, Yu T, Du R, Fan G, Liu Y, Liu Z, Xiang J et al (2020) Clinical course and risk factors for mortality of adult inpatients with COVID-19 in Wuhan, China: a retrospective cohort study. Lancet 395:1054-1062

4. Spiezia L, Boscolo A, Poletto F, Cerruti L, Tiberio I, Campello E, Navalesi P, Simioni P (2020) COVID-19 -related severe hypercoagulability in patients admitted to Intensive Care Unit for acute respiratory failure. Thromb Haemost. https://doi. org/10.1055/s-0040-1710018

5. Levi M, Van der Poll T (2017) Coagulation and sepsis. Thromb Res 149:38-44

6. Gupta N, Zhao YY, Evans CE (2019) The stimulation of thrombosis by hypoxia. Thromb Res 181:77-83

7. Luo W, Yu H, Gou J, Li X, Sun Y, Li J, Liu L (2020) Clinical pathology of critical patient with novel coronavirus pneumonia (COVID-19). Preprints, p 2020020407

8. Shanghai Clinical Treatment Expert Group for COVID-19 (2020) Comprehensive treatment and management of coronavirus disease 2019: expert consensus statement from Shanghai. Chin J Infect, p 38 (in Chinese)

9. Chong PY, Chui P, Ling AE, Franks TJ, Tai DY, Leo YS, Kaw GJ et al (2004) Analysis of deaths during the severe acute respiratory syndrome (SARS) epidemic in Singapore: challenges in determining a SARS diagnosis. Arch Pathol Lab Med 128:195-204

10. Bikdeli B, Madhavan MV, Jimenez D, Chuich T, Dreyfus I, Driggin E, Nigoghossian C, Ageno W, Madjid M et al (2020) COVID19 and thrombotic or thromboembolic disease: implications for prevention, antithrombotic therapy, and follow-up. J Am Coll Cardiol. https://doi.org/10.1016/j.jacc.2020.04.031

Publisher's Note Springer Nature remains neutral with regard to jurisdictional claims in published maps and institutional affiliations. 